\title{
A New View of the Origin of the Solar Wind
}

\author{
Richard Woo ${ }^{1}$ and Shadia Rifai Habbal ${ }^{2}$
}

\author{
'Jet Propulsion Laboratory, California Institute of Technology, Pasadena, California 91109 \\ 'Harvard-Smithsonian Center for Astrophysics, Cambridge, Massachusetts 02138
}

\begin{abstract}
This paper uses white-light measurements made by the SOHO LASCO coronagraph and HAO Mauna Loa Mk III K-coronameter to illustrate the new view of solar wind structure deduced originally from radio occultation measurements. It is shown that the density profile closest to the Sun at 1.15 Ro, representing the imprint of the Sun, is carried essentially radially into interplanetary space by small-scale raylike structures that permeate the solar corona and which have only been observed by radio occultation measurements. The only exception is the small volume of interplanetary space occupied by the heliospheric plasma sheet that evolves from coronal streamers within a few solar radii of the Sun. The radial preservation of the density profile also implies that a significant fraction of field lines which extend into interplanetary space originate from the quiet Sun, and are indistinguishable in character from those emanating from polar coronal holes. The white-light measurements dispel the long-held belief that the boundaries of polar coronal holes diverge significantly, and further support the view originally proposed in (1) that the fast solar wind originates from the quiet Sun as well as polar coronal holes.
\end{abstract}

\section{INTRODUCTION}

Our notions and understanding of the origin of the solar wind stem from remote sensing measurements of the Sun on the one hand, and direct observations of the solar wind by interplanetary spacecraft on the other, with a conspicuous absence of definitive measurements of the outer corona and intervening solar wind. Since high speed streams were first related to coronal holes over two decades ago, coronal holes have been thought to be the sole sources of the fast solar wind (2-4). As details of density and velocity structure in the outer corona have become available, first from radio occultation $(1,5,6)$, and subsequently from ultraviolet (7) and white-light measurements (8), a significantly different view of the source of the solar wind has emerged. A chronicle of the observational developments leading to this new view has been presented elsewhere (9), and will not be repeated here. Instead, we illustrate the new view with recent results on density structure from white-light measurements and their implications for the origin of the solar wind.

\section{IMPRINT OF THE SUN ON THE SOLAR WIND}

Shown in Figure 1 are the images of the Sun taken on August 11, 1997 comprising: (a) the Yohkoh soft X-ray full disk and the HAO Mauna Loa Mk III K-coronameter white-light images, (b) the Mauna Loa and the SOHO LASCO C2 white-light images, and (c) the SOHO LASCO C3 white-light image. We focus attention only on the northern hemisphere since the supporting pylon of the $\mathrm{C} 2$ and $\mathrm{C} 3$ external occulters is in the southeast. The northerm polar coronal hole, as projected on the solar disk and defined by the Kitt Peak He I $1083 \mathrm{~nm}$ map, is traced in yellow on the Yohkoh soft X-ray image in Figure la. The dashed radial yellow lines superimposed on the corona in Figures $\mathrm{I}$ a $-\mathrm{c}$ indicate the radial extensions of the $\mathrm{He} \mathrm{I}$ coronal hole boundaries in the plane of the sky, which are also represented by the black vertical lines on the latitudinal or azimuthal profiles of the white-light intensities in Figure 2.

The profiles in Figure 2, comprising measurements sampled once per degree of latitude, are displayed on a logarithmic vertical scale in order to show relative change and the limits of instrumental noise. The $\mathrm{pB}$ scans of the Mk III K-coronameter at heights of 1.15, 1.35, 1.45, and 1.55 Ro displayed in Figure 2a show that their range of variation rises steeply with increasing height - from a factor of 2-3 nearest the Sun at 1.15 Ro to an order of magnitude or higher for heights greater than $1.35 \mathrm{Ro}$. In spite of the steep rise in $\mathrm{pB}$ levels outside the coronal hole, the shapes of the $\mathrm{pB}$ profiles within the coronal hole remain essentially unchanged with increasing height. 
(a)
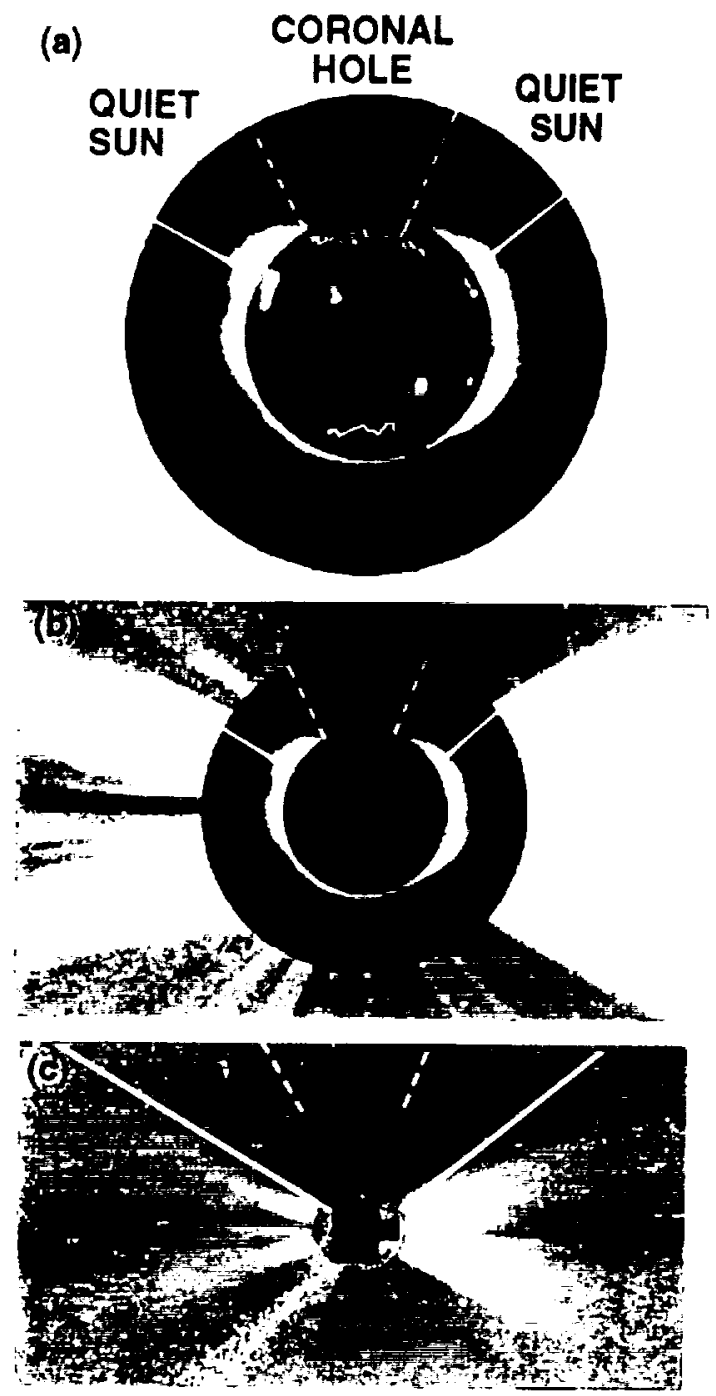

FIGURE 1. White-light observations of August 11, 1997. (a) Combined images of Yohkoh soft X-ray and Mk III Mauna Loa $\mathrm{K}$-coronameter $\mathrm{pB}$. The yellow line on the disk traces the coronal hole boundary as defined by the He I $1083 \mathrm{~nm}$ maps of the National Solar Observatory. (b) Combined images of SOHO LASCO C2 intensity and Mauna Loa pB. (c) Image of LASCO C3 intensity. The dashed yellow lines superimposed on the corona (and corresponding to the black vertical lines in Figure 2) indicate the radial extension of the polar coronal hole boundaries as defined by the He I $1083 \mathrm{~nm}$ maps; the solid yellow lines indicate the region over which there is coincidence between the density profiles at 1.15 and $5.5 \mathrm{Ro}$, as defined by the unshaded portions of the profiles in Figure $2 \mathrm{c}$.

However, the noisy profile at 1.55 Ro indicates that the measurements are only reliable closer than this distance to the Sun or when $\mathrm{pB}$ levels exceed $50 \times 10^{-10} \mathrm{Bo}$. The profile at 1.15 Ro has the strongest signal-to-noise ratio. Since the slight variations superimposed on these background density profiles represent the coronal rays or plumes seen in the corresponding images, measurement error is smaller than these variations.

The radial evolution of density structure in the corona is illustrated in Figure $2 \mathrm{~b}$. Here, the $\mathrm{pB}$ profiles of 1.35 and 1.45 Ro have been shifted so that their coronal hole profiles match that of the 1.15 Ro profile. To these profiles, we have added the $C 3$ intensity profile, whose vertical scale has been adjusted to match its coronal hole profile to the others. It is clear that density structure in the coronal hole is preserved with increasing heliocentric distance, but that the narowing of the streamers is accompanied by an increase in their brightness relative to that of the coronal hole. The most striking feature of the radial evolution of density structure is the large angular extent over which there is coincidence between the profiles nearest ( $1.15 \mathrm{Ro})$ and farthest from the Sun (5.5 Ro), as observed in the unshaded portion of the profiles in Figure 2c and also defined by the solid radial yellow lines in Figure 1. Remarkably, the coincidence extends well beyond the radial boundaries of the coronal hole, spanning $110^{\circ}$ in latitude (position angles $+60^{\circ}$ to $-50^{\circ}$ ). However, it is similar to that found when ranging or total electron content measurements at 20-30 Ro were compared with white-light measurements at 1.15 Ro (10). We have reproduced this result in Figure 3, which shows coincidence over a latitudinal range of $60^{\circ}$ in a quadrant of the sky.

As evident in Figure 1, the latitudinal range over which the density profile at 5.5 Ro is preserved encompasses as much of the quiet Sun as the polar coronal hole, with the east limb also including an active region. At $1.15 \mathrm{Ro}$, the quiet Sun dominates the density profile, with its corresponding $\mathrm{pB}$ level showing an increase of a factor of 2-3 over that of the coronal hole. At $5.5 \mathrm{Ro}$, the tapered coronal streamers, whose brightness is now at least an order of magnitude higher than that of the coronal hole, dominate the preserved profile, which can only be detected and defined by measurements with high sensitivity and high dynamic range, as in the case of the LASCO measurements (11). Early white-light measurements lacked these abilities, and the radial extension of the coronal hole boundary was not detected. Consequently, as illustrated in Figure 4, polar coronal holes were erroneously thought to evolve around the edges of the bright streamers and to diverge significantly $(4,12)$, rather than expand radially as is evident in the profiles of Figure 2.

The radially preserved imprint of the Sun at 1.15 Ro could only have reached 5.5 Ro via solar wind flowing along approximately radial open magnetic field lines originating in the quiet Sun as well as coronal holes. These field lines are associated with the ubiquitous low-density, low-contrast, small-scale (angular size smaller than a degree) raylike or filamentary structures that permeate the corona, and which have only been detected by radio 

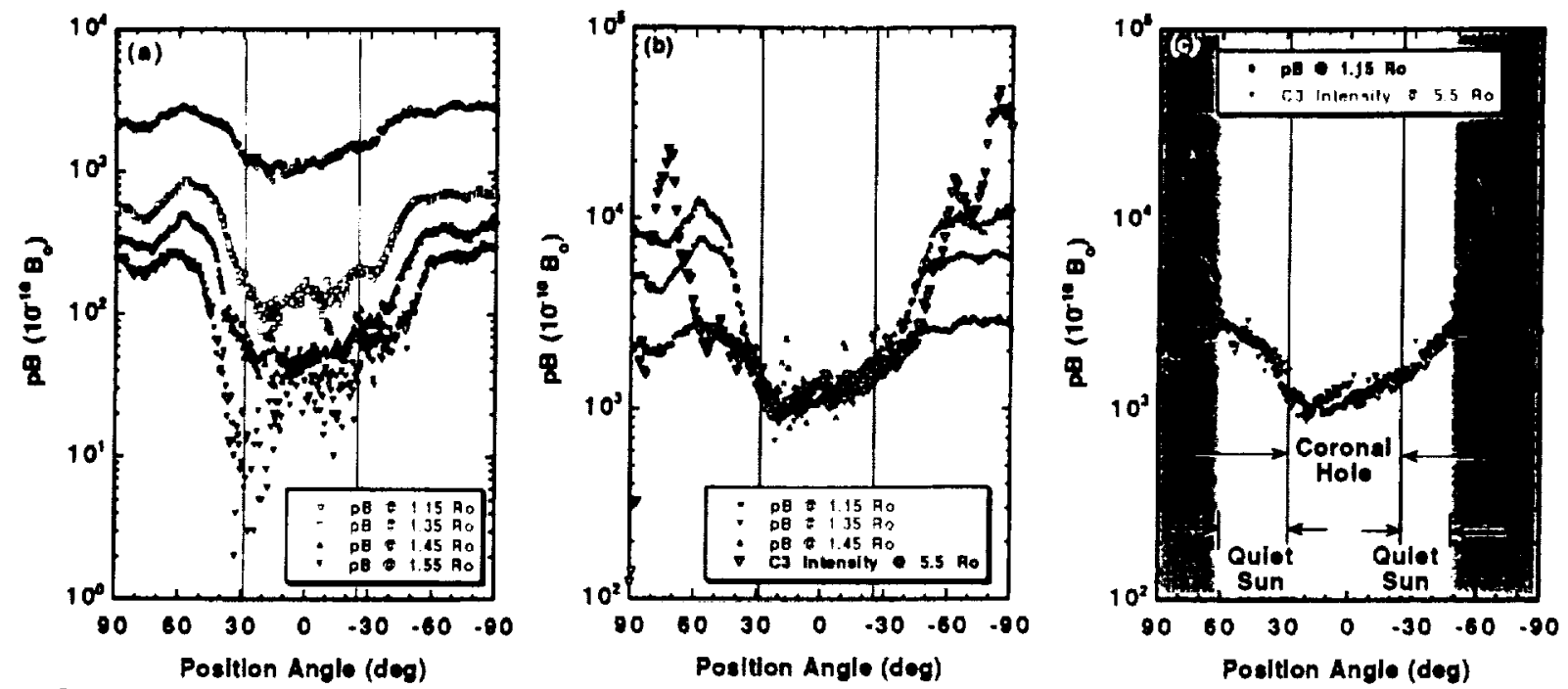

FIGURE 2. Latitudinal profiles of path-integrated density. The black vertical lines correspond to the coronal hole boundaries defined by the dashed yellow lines in Figure 1. (a) pB observed by Mk III Mauna Loa K-coronameter at altitudes of 1.15, 1.35, 1.45 , and 1.55 Ro. (b) Superimposed Mk III pB profiles at 1.15, 1.35, 1.45 Ro and SOHO C3 intensity profile at 5.5 Ro. (d) SOHO $\mathrm{C} 3$ intensity profile at 5.5 Ro superimposed on $\mathrm{Mk}$ III pB profile at $1.15 \mathrm{Ro}$. The unshaded portions of the profiles are where there is coincidence between the two.

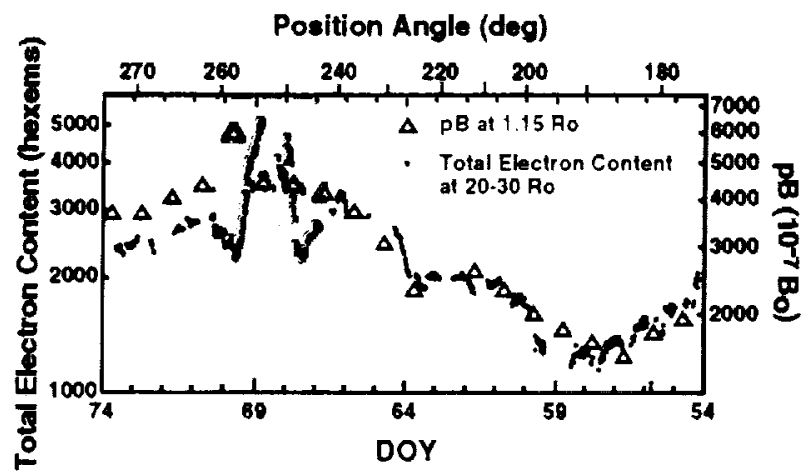

FIGURE 3. Time series of ranging or total electron content in hexems or $10^{16}$ electrons $/ \mathrm{m}^{2}$ in blue and Mauna Loa Mk III pB at 1.15 Ro in red as a function of day of year DOY in 1995. Position angle is indicated at the top of the figure. The two time series show good agreement except in the shaded region representing the evolved coronal streamer. Adapted from (10).

occultation measurements. The smallest density structures are at least two orders of magnitude smaller than those observed in white-light measurements, but are comparable to the smallest magnetic scales deduced indirectly from photospheric field measurements (13). While the presence of open magnetic fields in the quiet Sun appears to contradict the long held view that they are restricted to coronal holes, it is necessary to understand that coronal magnetic fields have not been measured directly, and that the traditional view is based solely on the impression of diverging polar coronal holes from white-light pictures, and theoretical magnetic field modeling only of large-scale structures $(14,15)$. Recent high spatial resolution eclipse measurements reproduced in Figure 5 (16) suggest that open field lines do indeed emerge from the quiet Sun.

The observed radial extension of the imprint of the Sun should not be confused with the impression of coronal evolution that one gets from a few individual coronal rays (also known as plumes) seen in (generally enhanced) white-light images $(17,18)$. The latter are observed in white-light pictures of coronal holes merely because they are the rays with the highest contrast, yet only produce at most $\pm 10 \%$ ripples on the background density profile. On the other hand, the background density profile or the imprint of the Sun varies by a factor of two across the coronal hole boundary, as is evident in Figure 2.

The latitudinal range of $110-120^{\circ}$ over which density structure is preserved is similar to that over which fast solar wind was observed inside 3 Ro by SOHO UVCS measurements (7), as well as far from the Sun by Ulysses in situ plasma (19). These combined results, therefore, imply that the fastest wind comes from the polar coronal holes, while the slightly slower fast wind at lower latitudes originates and flows along the open field lines from the quiet Sun, as discussed previously $(1,8,10)$.

\section{SMALL-SCALE RAYLIKE STRUCTURES}

The evidence for the radial extension of the imprint of the $S u n$ in both radio occultation and white-light measurements reinforces the close connection between these two types of measurements (20). Despite significant differences 

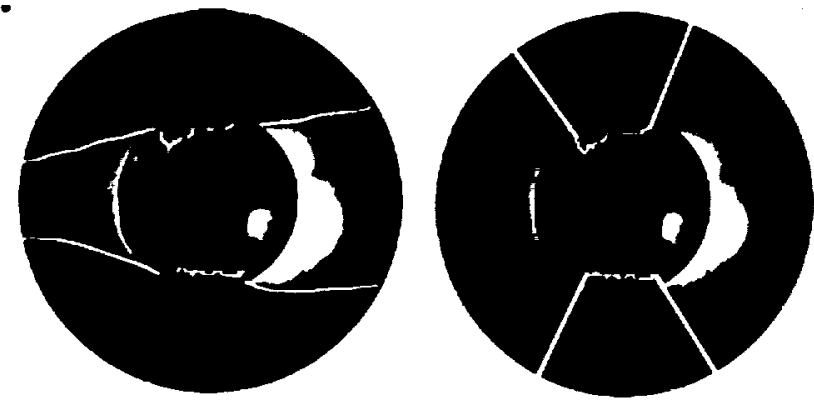

FIGURE 4. Combined images of Yohkoh soft X-ray and Mk III Mauna Loa K-coronameter pB of September 5, 1996, with yellow lines: (a) following the coronal streamer or apparent polar coronal hole boundary, (b) representing the radial extension of the polar coronal hole in (b). Adapted from (8).

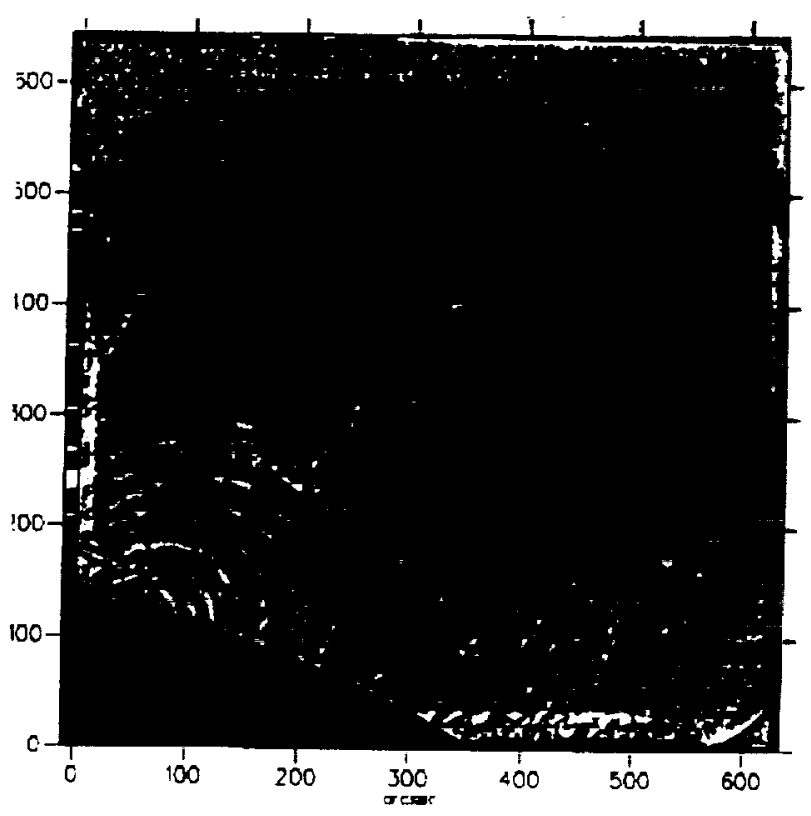

FIGURE 5. Enhanced high spatial resolution white-light image of corona during the total solar eclipse of July 11, 1991, showing dark and bright radial threads extending above a small prominence just above the limb. Reproduced from (16).

in spatial resolution, there are also similarities in their observations of small-scale features: raylike structures and inhomogeneities convected along with the solar wind.

Intensity scintillation observes only coronal structures that are smaller than the Fresnel size (21). Since the smallest structures are predominantly inhomogeneities that are convected along with the solar wind (5) (as illustrated in Figure 6a), by tracking the scattering from them with multiple stations, intensity scintillation measurements (commonly known as IPS for interplanetary scintillation) have served for many years as an invaluable means for indirectly measuring solar wind velocity $(22,23)$.
For a long time, it was thought that all small-scale structures that gave rise to radio propagation and scattering phenomena, including phase scintillation, represented convected inhomogeneities as depicted in Figure 6a. Angular broadening measurements near the Sun have exhibited strikingly high anisotropy (24), i.e., radio scattering that is higher in one direction than in the other, and an example of these measurements is reproduced in Figure $6 \mathrm{~b}(25)$. The observed high anisotropy has led to the revised picture as sketched in Figure 6c, showing a corona with high contrast raylike structures in addition to the low contrast inhomogeneities. The angular broadening measurement of Figure $6 \mathrm{~b}$ has been appropriately oriented and superimposed on this new picture to show that angular broadening is higher in the direction transverse to the raylike structures than in the direction parallel to them. This illustrates that the anisotropy is caused by the significantly high contrast (high density fluctuations producing the high radio scattering) across the raylike structures when compared with that in the radial direction and across the inhomogeneities.

Although white-light measurements such as those in Figure 1 have much lower spatial resolution, the images are dominated by raylike structures for the very same reason; the raylike structures have a significantly higher contrast than the convected inhomogeneities. With the outstanding sensitivity of the LASCO measurements, the convected inhomogeneities are now also being observed (26), and their tracking is yielding velocity estimates of the solar wind much in the same manner as IPS has. The angular broadening measurements, therefore, indicate that if the resolution of white-light measurements were to be improved by two orders of magnitude, the corona would still be strikingly dominated by raylike structures, as it is in Figure 1.

\section{CONCLUSION AND SUMMARY OF NEW VIEW}

The unprecedented sensitivity and dynamic range of the LASCO coronagraph (27) have made it possible to detect and observe faint structures in the outer corona that had not been observed in earlier white-light measurements. Global imaging of the corona using measurements by the LASCO coronagraph and $\mathrm{Mk}$ III K-coronameter illustrates the new view of coronal structure originally obtained from radio occultation measurements. The white-light measurements not only reinforce this new view and its implications for the origin and evolution of the fast solar wind, they also demonstrate how the raylike structures observed in white-light measurements are similar to the significantly smaller raylike structures observed by radio occultation measurements. 
(a)

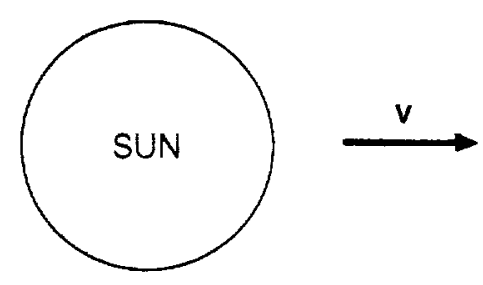

(b)

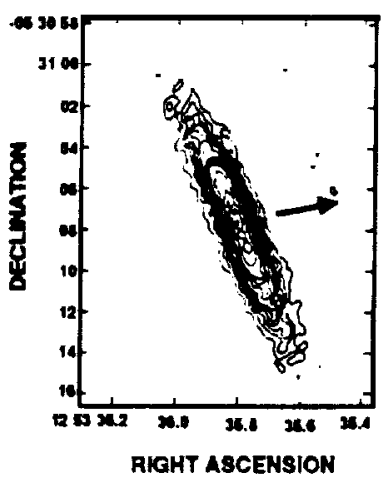

(c)

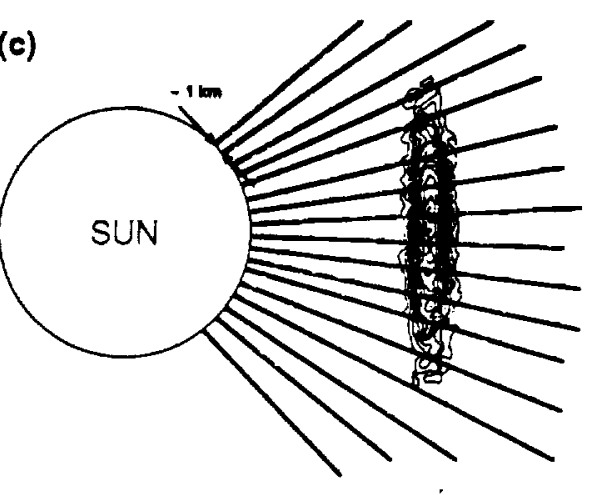

FIGURE 6. (a) Conceptual sketch of the solar corona showing convected inhomogeneities in the solar wind. (b) Image of angular broadened radio source reproduced from (16). (c) Revised conceptual sketch of the solar corona showing high contrast raylike structures in addition to the low contrast convected inhomogeneities in the solar wind with radio scattered image in (b) properly oriented and superimposed.

We have also shown how the fading detectability of the low contrast coronal hole boundary on the one hand, and the increasing contrast of the evolving coronal streamer boundary on the other, combined to produce the erroneous conclusion based on early white-light measurements that polar coronal holes underwent significant divergence as they extended into interplanetary space. Along with magnetic field modeling, this result in turn led to the idea that open field lines existed solely in coronal holes, and the natural conclusion that the high latitude fast wind observed by Ulysses in situ plasma measurements all came from polar coronal holes $(27,28)$.

The new view is conceptually simple. Two major components comprising large- and small-scale density structures give rise to the corona observed in white-light images, as depicted in Figure 7a. The new view is most readily revealed in path-integrated measurements near solar minimum when the tilt in the solar magnetic dipole is minimum and the heliospheric current sheet is flat. In this configuration, the two major components of density structures are most isolated from each other. Coronal streamers, the brightest and most dominant features nearest the Sun, represent the large-scale structures summarized in Figure $7 \mathrm{~b}$. Evolving into the heliospheric plasma sheet within a few solar radii of the Sun (29), the narrow and bright streamer stalks ironically occupy only a small volume of interplanetary space (30), but carry the slowest solar wind (7). Small-scale raylike structures, which originate from all over the Sun, and pervade the solar corona as pictured in Figure 7c, represent the second major component of density structure. These low-density, low-contrast, small-scale structures are readily detected in radio occultation measurements but are not observed in white-light images. Fast wind flows along these small-scale structures which extend from coronal holes as well as thread their way between/through closed field regions of the quiet Sun (as seen in Figure 7a), and carry the imprint of the Sun essentially radially into interplanetary space. The most conspicuous feature of the imprint is the coronal hole signature, as shown in Figure 7d.

\section{ACKNOWLEDGMENTS}

It is a pleasure to thank $R$. Howard (NRL) for many useful discussions about the LASCO measurements and their capabilities. We are also grateful to J.W. Armstrong for discussions, $C$. Copeland for producing the paper and the figures, J. Burkepile and H. Higgins of HAO for generously providing the Mauna Loa Mk III K-coronameter (operated by NCAR/HAO) data. The NSO/Kitt Peak He I $1083 \mathrm{~nm}$ data used here are produced cooperatively by NSF/NOAO, NASA/GSFC, and NOAA/SEL. We thank the Yohkoh Data Archive Center for the Yohkoh soft Xray images. This paper describes research carried out at the Jet Propulsion Laboratory, Califomia Institute of Technology, under a contract with the National Aeronautics and Space Administration. Support for S.R. Habbal was provided by NASA grant NAG5-6215. LASCO was constructed and is operated by an intemational consortium consisting of the Naval Research Laboratory (Washington, DC), the Department of Space Research at the University of Birmingham (Birmingham, United Kingdom), 

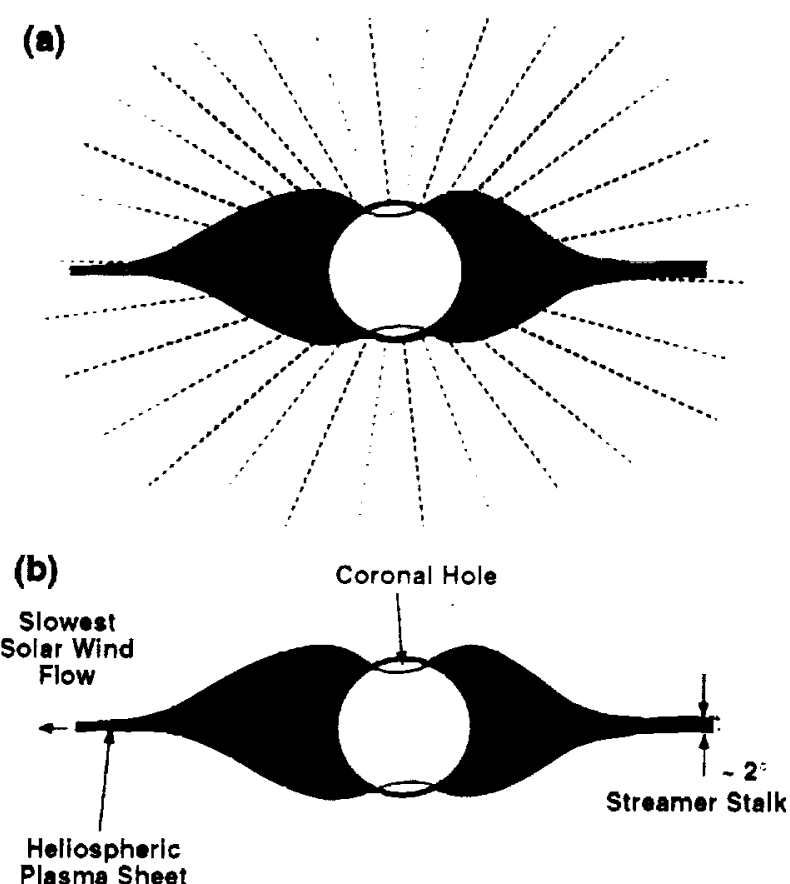

(c)

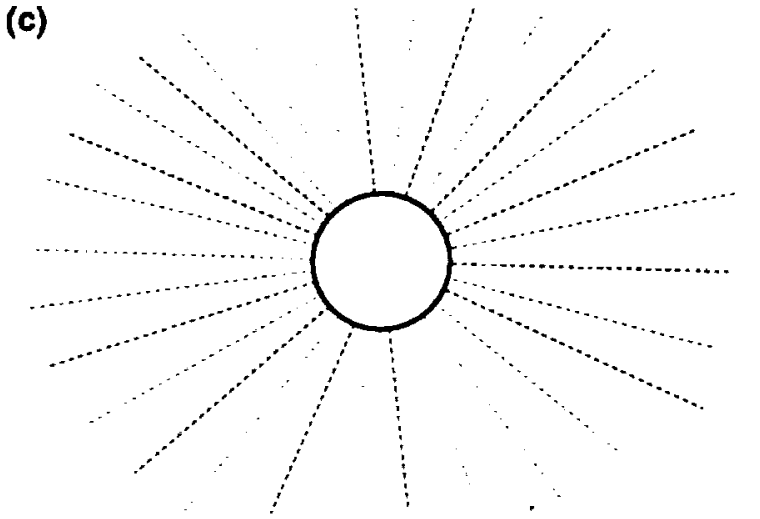

(d)

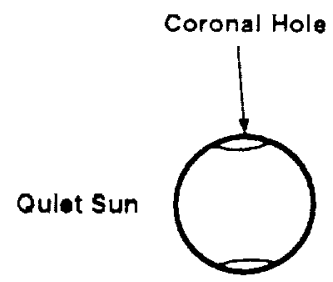

FIGURE 7. Conceptual sketches of: (a) new view of the solar wind, (b) large-scale coronal streamers, (c) ubiquitous low contrast raylike structures, and (d) collective brightness of raylike structures. the Max-Planck Institute for Aeronomy (Lindau, Germany) and the Laboratory for Space Astronomy (Marseilles, France). SOHO is a mission of international cooperation between ESA and NASA.

\section{REFERENCES}

1. Woo, R., and Habbal, S.R., Geophys. Res. Lett. 24, 11591162 (1997).

2. Krieger, A., et al., Solar Phys. 29, 505 (1973).

3. Hundhausen, A., in Coronal Holes and High Speed Streams, Colorado Associated University Press, Boulder, 1977, pp. 225-329.

4. Zirker, J.B., ed., Coronal Holes and High Speed Wind Streams, Colorado Associated University Press, Boulder, 1977.

5. Woo, R., Nature 379, 321-322 (1996).

6. Woo, R., and Martin, J.M., Geophys. Res. Lett. 24, 25352538 (1997).

7. Habbal, S.R., et al., Astrophys. J. 489, L103-106 (1997.

8. Woo, R., et al., Astrophys. J. 513, (1999).

9. Woo, R., and Habbal, S.R., Multiscale filamentary structures in the solar corona and their implications for the origin and evolution of the solar wind, in Physics of Space Plasmas (1998). Number 15, eds. T. Chang and J.R. Jasperse, MIT Center for Theoretical Geo/Cosmo Plasma Physics, Cambridge, MA, 1998.

10. Woo, R., and Habbal, S.R., Astrophys. J. 510, L69-L72 (1999).

11. Brueckner, G.E., et al., Solar Phys. 162, 357-402 (1995).

12. Munro, R.H., and Jackson, B.V., Astrophys. J. 213, 874-886 (1977).

13. Sanchez Almeida, J., Astrophys. J. 491, 993-1008 (1997).

14. Schatten, K.H., et al., Solar Phys. 6, 442-455 (1969).

15. Pneuman, G.W., and Kopp, R.A., Solar Phys. 18, 258-270 (1971).

16. November, L.J., and Koutchmy, S., Astrophys. J. 466, 512528 (1996).

17. Guhathakurta, M., and Fisher, R., Geophys. Res. Lett. 22, 1841-1844 (1995).

18. DeForest, C.E., et al., Solar Phys. 175, 393-410 (1997).

19. McComas, D.J., et al., Geophys. Res. Letts. 25, 1-4 (1998).

20. Woo, R., Coronal structures observed by radio propagation measurements, in Solar Wind Eight, eds. D. Winterhalter, J. Gosling, S. Habbal, W. Kurth and M. Neugebauer, New York, AIP CP 382, 1996, pp.38-43.

21. Ishimaru, A., Wave Propagation and Scattering in Random Media, Vol. 2, Academic Press, New York, 1978, p. 360.

22. Grall, R.R., et al., Nature 379, 429-432 (1996).

23. Kojima, M., et al., J. Geophys. Res. 103, 1981-1989 (1998).

24. Armstrong, J.W., et al., Astrophys. J. 358, 685-692 (1990).

25. Narayan, R., et al., MNRAS 241, 403-413 (1989).

26. Sheeley, Jr., N.R., et al., Astrophys. J. 484, 472-478 (1997).

27. Gosling, J.T., et al., Geophys. Res. Lett. 22, 3329-3332 (1995).

28. Geiss, J., et al., Science 268, 1033-1036 (1995).

29. Bavassano, B., et al., Geophys. Res. Lett. 24, 1655-1658 (1997).

30. Wang, Y.-M., et al., Astrophys. J. 485, 875-889 (1997). 